\title{
Interaction between systolic and diastolic time intervals in atrial fibrillation
}

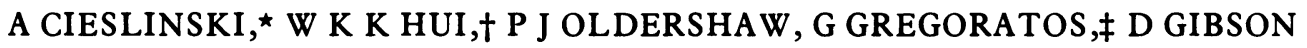 \\ From the Department of Cardiology, Brompton Hospital, London
}

SUMmaRY To assess determinants of left ventricular filling time at rest simultaneous phonocardiograms, $M$ mode echocardiograms, and indirect carotid pulse traces were performed on 50 patients with atrial fibrillation. Thus the effect of random variation in $R R$ interval in the absence of atrial contraction on the inter-relation between systolic and diastolic events could be seen. Left ventricular filling time was closely related to $R R$ interval and affected by it to a much greater extent than ejection time, so that filling time became very short when heart rate was fast. Two mechanisms were, however, identified whereby filling time was preserved: (a) prolongation of filling during the preejection period of the following cycle, so that when the $R R$ interval was less than $400 \mathrm{~ms}$ all of ventricular filling frequently occurred at this time; and (b) abbreviation of the $\mathrm{QA}_{2}$ interval of the succeeding beat with reduced filling, so that mitral valve opening occurred earlier. Changes in the duration of isovolumic contraction or relaxation time were not involved, neither could alteration in left ventricular contractile state resulting from variation in the interval between beats be shown to affect filling time at rest.

The striking sensitivity of left ventricular filling time to rapid heart rate in atrial fibrillation suggests that it may be a major factor limiting cardiac performance. Diastolic as well as systolic events should, therefore, be taken into account when the effects of drugs or other physiological manoeuvres are studied in these patients.

The assessment of myocardial performance by measuring time intervals between events occurring in the cardiac cycle has a long history. In the midnineteenth century Marey first described the sphygmograph which enabled left ventricular ejection time to be derived from externally recorded arterial pulse tracings. With the invention of the electrocardiogram and phonocardiogram at the turn of the century interest in systolic time intervals pioneered by the classic works of Garrod, ${ }^{1}$ Wiggers, ${ }^{2}$ and Katz and Feil ${ }^{3}$ laid the foundation for the use of this method as a valuable non-invasive tool for assessing left ventricular systolic function. ${ }^{4}$ The relation between left ventricular ejection time and heart rate and its behaviour in different

Requests for reprints to Dr Derek Gibson, Cardiac Department, Brompton Hospital, Fulham Road, London SW3 6HP.

Present addresses:

*Institute of Cardiology, School of Medicine, Poznan, Poland.

†Department of Medicine, Queen Mary Hospital, University of Hong Kong. ¥Cardiology Division, University of California Medical Center, San Diego, California, USA.

Accepted for publication 10 November 1983 diseases have all been extensively studied. ${ }^{5}$ Nevertheless, interest in the time intervals of systole has not been balanced by a similar interest in those of diastole. In particular the diastolic counterpart of ejection time, the left ventricular filling period or "injection time," has received but scant attention, ${ }^{6}$ although a close relation must exist between ejection and filling since what comes out of the left ventricle must first go in.

In the present study we selected a group of patients with atrial fibrillation, taking advantage of the varying cycle lengths in the resting state which allows a large number of beats at different $R R$ intervals to be analysed from a single recording, in order to investigate the intricate relation between ejection and filling using a simple external recording technique based on the $M$ mode echo.

\section{Patients and methods}

Fifty patients in atrial fibrillation (20 men and 30 women) were studied. Their ages ranged from 24 to 88 years. Fourteen had atrial fibrillation with a normal 


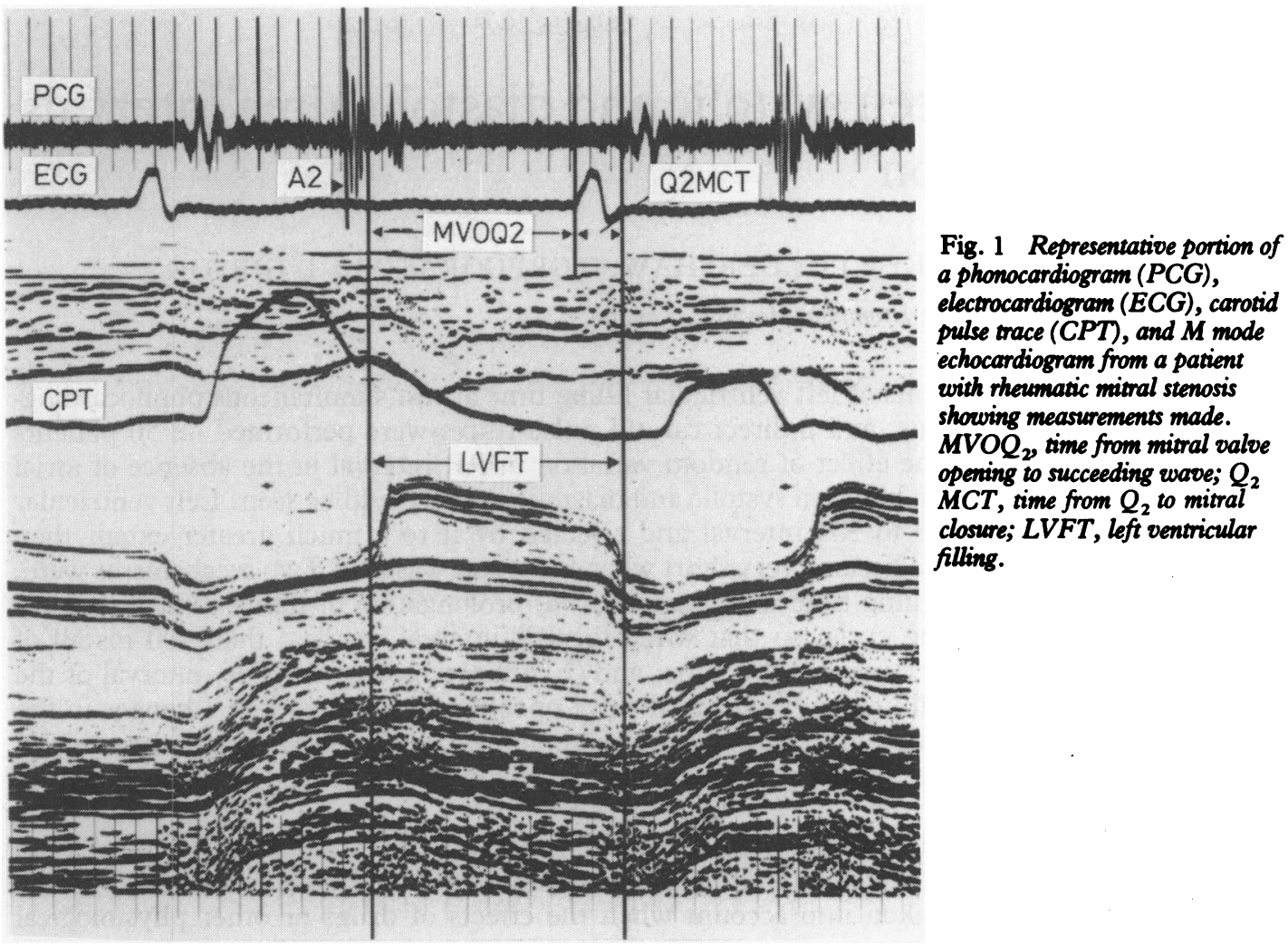

mitral valve and were mainly patients with congestive cardiomyopathy; one patient, however, had mitral valve prolapse without regurgitation. Nine had mitral valve replacement (Starr-Edwards or Björk-Shiley), 16 pure rheumatic mitral stenosis, nine rheumatic mitral stenosis and regurgitation, and two nonrheumatic mitral regurgitation. None had significant aortic regurgitation, and all but four were taking a digitalis preparation when the study was carried out.

$M$ mode echocardiograms were recorded in the resting state with patients in a $30^{\circ}$ left lateral position using a Cambridge Instruments Ultrasonoscope (frequency $2.25 \mathrm{MHz}$, repetition rate $1000 / \mathrm{s}$ ). The direction of the ultrasonic beam was adjusted so that the tips of both leaflets of the mitral valve or, in the case of patients with mechanical mitral prostheses, the echoes from the leading edge of the ball or disc were clearly shown. Simultaneous recordings of the external carotid pulse (using a Cambridge Instruments transducer, time constant $4 \mathrm{~s}$, lower frequency limit $0.05 \mathrm{~Hz}$ ), phonocardiogram (using a Leatham microphone and a medium frequency filter applied at the area at which the aortic component of the second sound was most easily heard), and the standard lead II electrocardiogram were made. The output was dis- played on a Cambridge Instruments strip chart recorder at a paper speed of $100 \mathrm{~mm} / \mathrm{s}$ (Fig. 1). Only normally conducted beats were analysed. Ventricular extrasystoles and post-extrasystolic pauses were excluded as were all patients with QRS duration exceeding $110 \mathrm{~ms}$. Twenty to 30 consecutive beats were analysed for each patient and the following time intervals, rounded to the nearest $5 \mathrm{~ms}$, were measured directly from the strip chart for each cardiac cycle: (a) RR interval; (b) $Q$ to mitral valve opening (QMVO); (c) left ventricular filling time (LVFT) measured as the time interval from the onset of mitral valve opening to mitral valve leaflet coaptation (MCT) at the onset of the succeeding systole; (d) total electromechanical systole $\left(\mathrm{QA}_{2}\right)$-measured from the $Q$ wave of the electrocardiogram to the first high frequency vibration of the aortic component of the second heart sound (the precise timing of $A_{2}$ was confirmed by recording simultaneously the aortic valve echogram in each patient); and (e) left ventricular ejection time (LVET)-measured from the onset of the rapid upstroke of the carotid pulse to the trough of the incisura.

The following values were derived from the above measurements: (a) isovolumic relaxation time- 


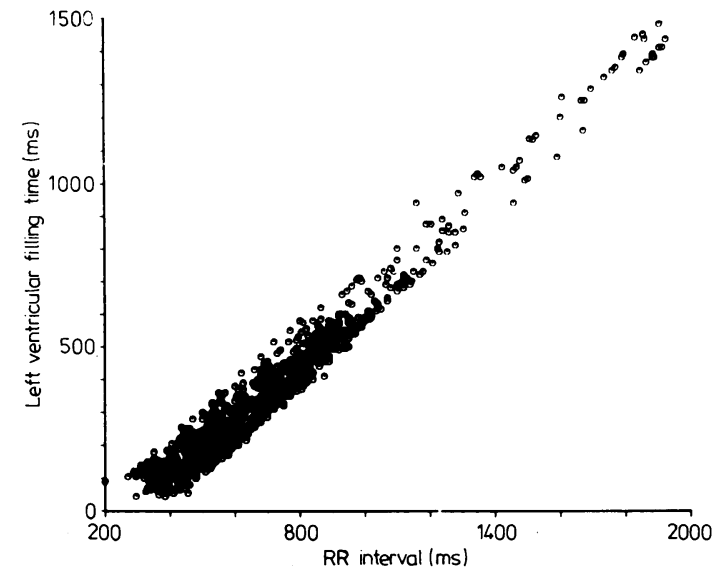

Fig. 2 Left ventricular filling time plotted against cycle length $(R R)$. The linear relation and extent of correlation are evident.

calculated as QMVO- $\mathrm{QA}_{2}$; (b) time from mitral valve opening to the start of the succeeding $Q$ wave $\mathrm{Q}_{2}\left(\mathrm{MVOQ}_{2}\right)$-calculated as $\mathrm{RR}-\mathrm{QMVO}$, and hence the period of left ventricular filling occurring after the $Q$ wave of the following cardiac cycle $\left(Q_{2} M C T\right)$ as LVFT-MVOQ ${ }_{2}$; (c) pre-ejection period (PEP)calculated as $\mathrm{QA}_{2}-\mathrm{LVET}^{7}$; and (d) isovolumic contraction time (IVC)-calculated as PEP- $\mathrm{Q}_{2}$ MCT (of the preceding beat).

\section{STATISTICAL METHODS}

Correlation coefficients were calculated by the method of least squares. Differences between groups were assessed using either Student's $t$ test or the MannWhitney U test.

\section{Results}

A total of 1386 cardiac cycles was analysed, giving a mean of 28 beats per patient.

\section{LEFT VENTRICULAR FILLING TIME}

All patients

The range through which left ventricular filling time varied with the $R R$ interval was great (from $50 \mathrm{~ms}$ to a

Table 1 Variation in $Q_{2} M C T$ interval (means $\pm S D$ ) with cycle length

\begin{tabular}{lll}
\hline $\begin{array}{l}R R \text { interval } \\
(m s)\end{array}$ & $\begin{array}{l}Q_{2} M C T \text { interval } \\
(m s)\end{array}$ & No of cycles analysed \\
\hline$<400$ & $84 \pm 20$ & 118 \\
$400-600$ & $73 \pm 17^{\star}$ & 441 \\
$600-800$ & $70 \pm 17^{\star \star}$ & 458 \\
$800-1000$ & $70 \pm 16$ & 254 \\
$>1000$ & $67 \pm 13$ & 115 \\
\hline
\end{tabular}

${ }^{\star} \mathrm{p}<0.001$ vs $\mathrm{RR}<400,{ }^{\star \star} \mathrm{p}<0.01$ vs RR 400-600.

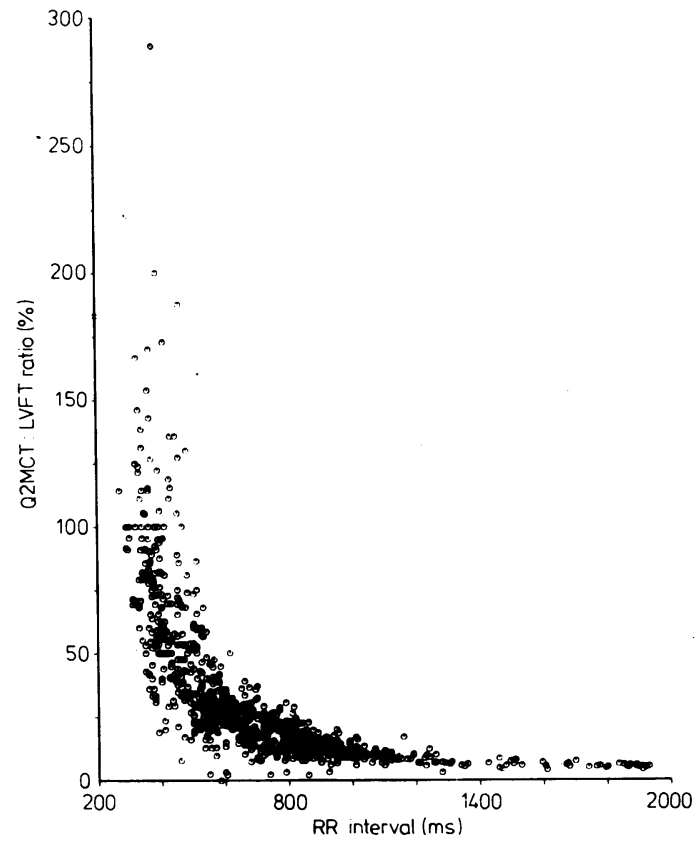

Fig. 3 Plot of the ratio of $Q_{2} M C T$ to left ventricular filling time (LVFT) against cycle length. As the $R R$ interval decreases to less than 400 ms the $Q_{2} M C T$ : LVFT frequently exceeds $100 \%$.

high of $1645 \mathrm{~ms}$ ). A positive linear relation existed between total filling time and the corresponding cycle length in all patients studied. Correlation coefficients ranged from 0.932 to 0.999 (mean of 0.984 ); the regression equation describing this relation for the whole group was $L V F T=0.834(R R)-219.0 ; S E E \pm 48$ ms (Fig. 2). Correlation was significantly lower in patients whose mean cycle length was less than $600 \mathrm{~ms}$ $(0.969 \pm 0.018)$ but approached unity when the mean cycle length was more than $1000 \mathrm{~ms}(0.998 \pm 0.001)$ The regression equation predicted that the mitral valve would fail to open (LVFT $=0$ ) at $R R$ intervals of less than $264 \pm 48 \mathrm{~ms}$ suggesting that, in atrial fibrillation at least, a minimum cycle length is necessary for ventricular filling to take place.

The time interval $Q_{2}$ MCT increased to $84 \pm 20 \mathrm{~ms}$ when the RR interval was less than $400 \mathrm{~ms}$ from $67 \pm 13 \mathrm{~ms}$ when $\mathrm{RR}$ was more than $1000 \mathrm{~ms}$ (Table 1), although it changed little within the more physiological range of heart rates. Not surprisingly, $\mathrm{Q}_{2} \mathrm{MCT}$ as a percentage of the filling time bore an inverse relation to cycle length (Fig. 3), so much so that at $R R$ intervals below $\mathbf{4 0 0} \mathrm{ms}$ this ratio frequently exceeded $100 \%$, with mitral valve opening occurring after the onset of the $Q$ wave of the next beat (Fig. 4). The importance of the $Q_{2}$ MCT period becomes obvi- 


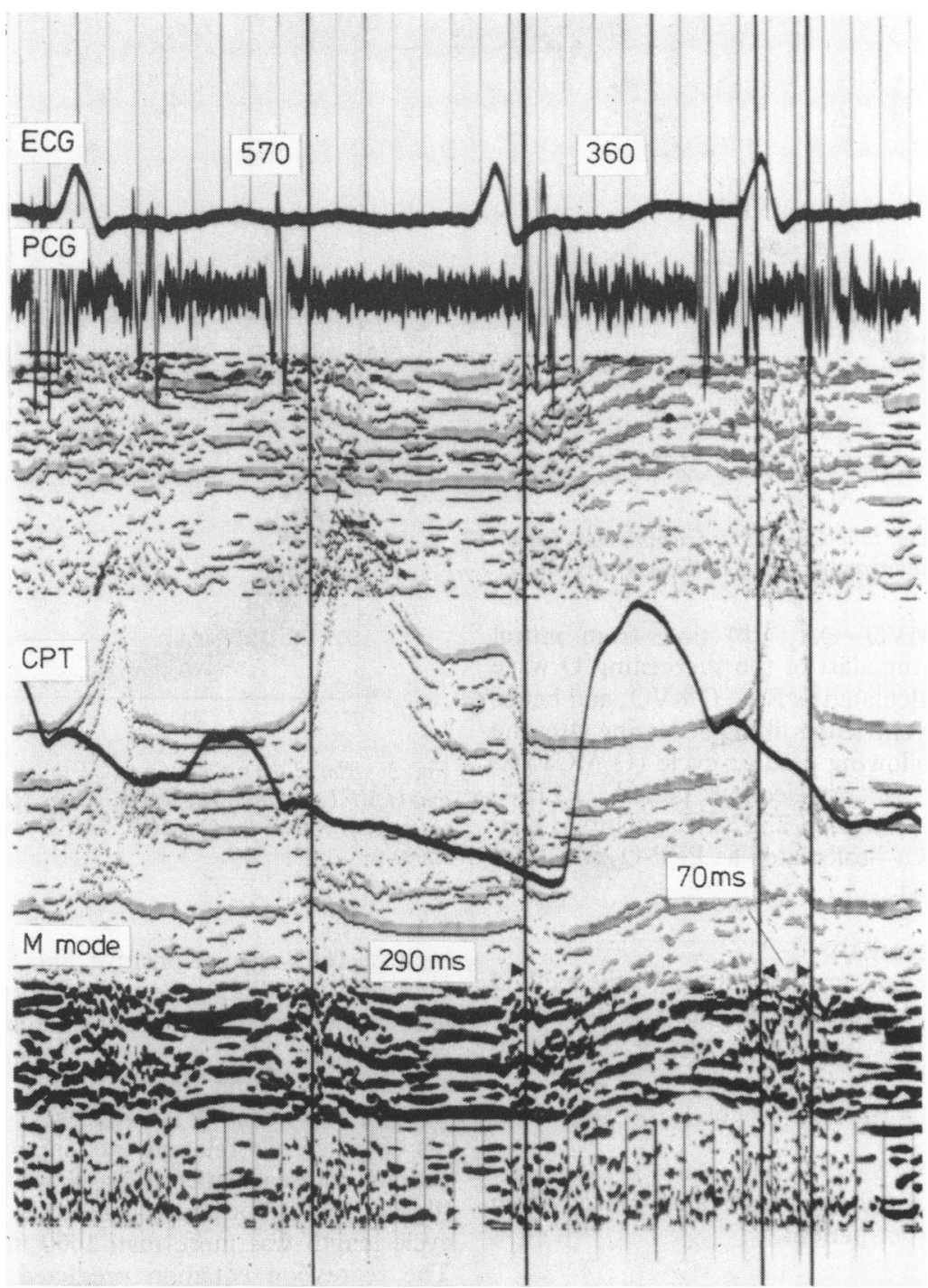

Fig. 4 Tracings showing that during a short cycle (RR interval $360 \mathrm{~ms}$ ) the mitral valve opens after the onset of the succeeding $Q R S$ and the entire left ventricular filling time of 70 ms takes place during the pre-ejection period of the next beat.

ous when it is considered that in all 1386 cycles analysed the mitral valve remained open until well after the onset of $Q_{2}$ ( $Q$ of the following cycle), indicating that this "systolic" period is available for continued ventricular filling even in the absence of atrial contraction.

\section{DIFFERENT DISEASE GROUPS}

Correlation between ventricular filling time and $R R$ interval in patients with different underlying disease entities did not seem to vary, except for a slight differ- ence between patients with normal mitral valves and mitral valve prostheses. The slopes of the regression lines for filling time $v s R R$ interval were again not significantly different between groups.

The same applies to the zero intercept-that is, the predicted $R R$ interval at which the mitral valve failed to open. The only exception was a slightly longer value of $R R$ interval for the group with mitral prostheses than for those with atrial fibrillation alone, which just reached statistical significance. This finding suggests that when inflow is impeded by the 

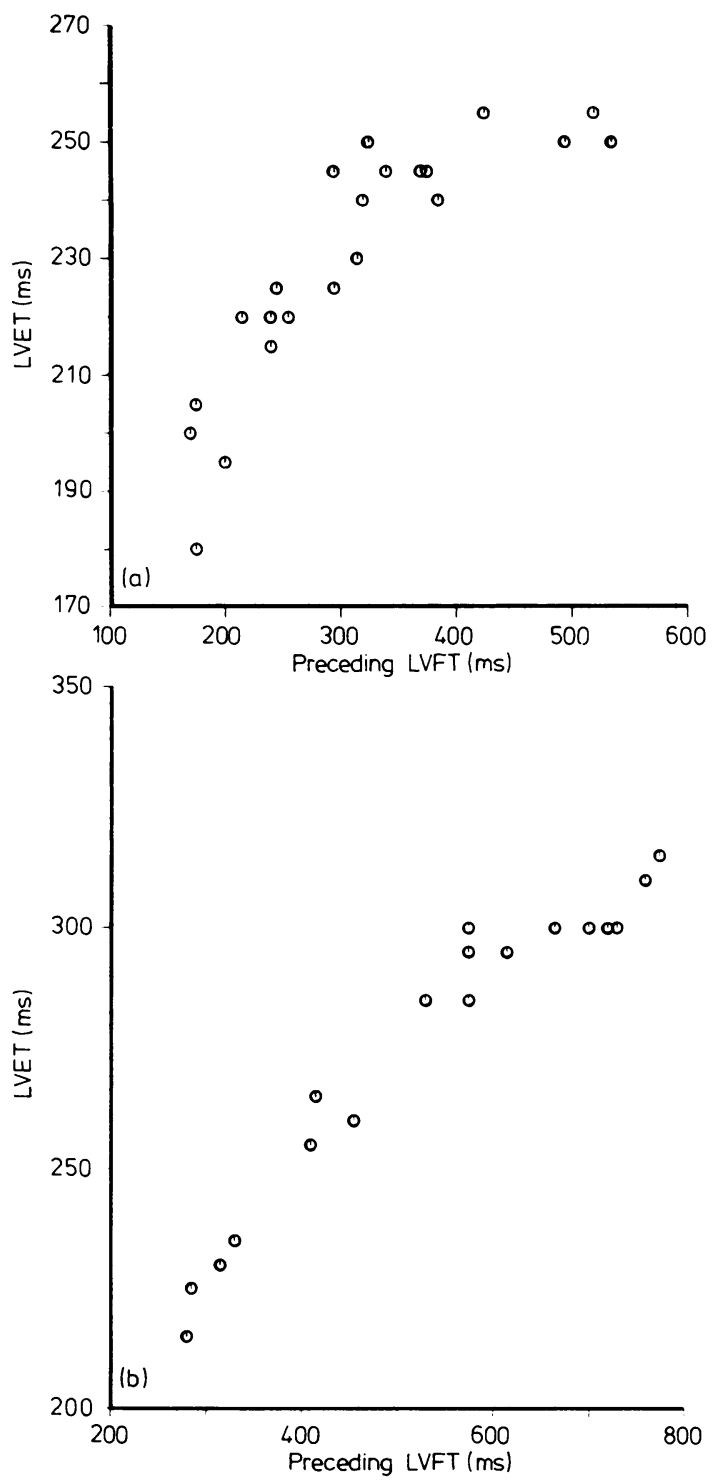

Fig. 5 Plots of left ventricular ejection time (LVET) against the preceding cycle left ventricular filling time (LVFT) in two different patients. The relation is discontinuous. (a) In the patient with a normal mitral valve and lone atrial fibrillation the transition point of the curve occurs at approximately $400 \mathrm{~ms}$. (b) In the the presence of mitral stenosis the transition is delayed.

inertia of the prosthetic valve ball, in the face of normal left atrial pressure, a somewhat longer cycle length is necessary for mitral valve opening.

ISOVOLUMIC RELAXATION TIME

Isovolumic relaxation time varied within a narrow
Table 2 Values of pre-ejection and isovolumic contraction periods at various preceding $R R$ intervals. Figures are means $\pm S D$ (number of observations in parentheses)

\begin{tabular}{lll}
\hline $\begin{array}{l}\text { Preceding } R R \\
\text { interval }\end{array}$ & Pre-ejection period & $\begin{array}{l}\text { Isovolumic contraction } \\
\text { time }\end{array}$ \\
\hline$<400$ & $126 \pm 42(42)$ & $40 \pm 19(36)$ \\
$400-600$ & $116 \pm 24(222)$ & $43 \pm 21(197)$ \\
$600-800$ & $118 \pm 27(241)$ & $47 \pm 20(218)$ \\
$800-1000$ & $113 \pm 24(122)$ & $44 \pm 15(110)$ \\
$>1000$ & $107 \pm 15(70)$ & $39 \pm 17(67)$ \\
\hline
\end{tabular}

range (mean $45 \mathrm{~ms}$ ) in individual patients despite the changing cycle lengths. No significant correlation was found between the relaxation time and the corresponding or preceding $R R$ intervals.

$\mathrm{QA}_{2}$ INTERVAL

Variation in the $\mathrm{QA}_{2}$ interval in individual patients was small compared with that in the left ventricular filling time, with a mean range of only $45 \mathrm{~ms}$. $\mathrm{QA}_{2}$ was related to the cycle length of the preceding beat in a curvilinear fashion, being directly proportional when the preceding RR interval was short and becoming independent as it lengthened. The point at which this transition occurred varied with different disease groups, occurring at longer preceding $R R$ intervals in patients with left ventricular inflow obstruction (rheumatic mitral valve disease and mitral prostheses groups) than in those in whom the mitral valve was normal.

\section{LEFT VENTRICULAR EJECTION TIME}

In general left ventricular ejection time had a similar curvilinear relation to the preceding cycle length as $\mathrm{QA}_{2}$, but the range over which it varied was greater (mean $80 \mathrm{~ms}$ ), and the transition point of the curve was reached at longer preceding RR intervals. An identical relation existed between ejection time and filling time of the preceding beat (Fig. 5a). Again, as in the case of $\mathrm{QA}_{2}$, in patients with left ventricular inflow obstruction the transition point occurred at longer preceding cycle lengths than in patients in whom the mitral valve was normal (Fig. 5b).

\section{PRE-EJECTION PERIOD AND ISOVOLUMIC CONTRACTION TIME}

The pre-ejection period varied over a range of about $50 \mathrm{~ms}$ in individual patients. It shared an inverse relation to the cycle length of the previous beat, in parallel to that of $\mathrm{Q}_{2}$ MCT which constitutes its first part. For the whole group its mean value decreased only from 126 to 107 ms over the range of measured RR intervals (Table 2), a difference of less than $20 \mathrm{~ms}$. On the other hand, isovolumic contraction time remained relatively constant at approximately $\mathbf{4 0} \mathrm{ms}$ through the entire range of preceding $R R$ intervals (Table 2 ). 


\section{Discussion}

Atrial fibrillation provides a unique opportunity for studying the complex interplay between left ventricular ejection and filling over a large number of randomly associated cycle lengths, free from the influence of other changes in physiological state. Our results show a highly significant relation between left ventricular filling time and cycle length, with correlation coefficients approaching unity. It is, of course, obvious intuitively that some such correlation must exist, since if $R R$ intervals are indeed random or nearly so the onset of each succeeding systole will be virtually independent of preceding systolic events although having the potential to influence subsequent ones. Nevertheless, the magnitude of the correlation coefficients was unexpected, suggesting that the exact timing of both mitral valve opening and closure were highly non-random, and indeed both proved worthy of further study.

Ventricular filling in a patient with atrial fibrillation ends with the onset of the succeeding ventricular systole. Nevertheless, we find considerable beat to beat variation in the exact time that it did so with respect to the onset of the QRS complex of the succeeding beat. When the RR interval was greater than $1000 \mathrm{~ms}$ the interval $Q_{2}$ to mitral valve closure $\left(Q_{2} M C T\right)$ was approximately $65 \mathrm{~ms}$, but this increased to $85 \mathrm{~ms}$ or more when the RR interval was less than $400 \mathrm{~ms}$. This additional period of ventricular filling is probably of little physiological importance when diastole is long, particularly as rapid filling is virtually complete by $100 \mathrm{~ms}$ after mitral valve opening. ${ }^{8} \mathrm{As}$ the $\mathrm{RR}$ interval decreases, however, this late component of filling becomes progressively more important, and when ventricular rate is rapid all filling may occur after the onset of the $Q$ wave of the succeeding beat (Fig. 4). Indeed, when the $R R$ interval was in the range 200$300 \mathrm{~ms}$ the time available for filling was less even than this, falling in some cases to as little as one half of this period. Such delay in mitral valve closure with respect to the onset of the succeeding QRS complex has several implications. It represents a mechanism by which filling time is preserved when $R R$ interval is short. In addition, since electromechanical delay varies little under widely different physiological conditions 9 delayed mitral valve closure also indicates that under suitable conditions left ventricular filling can continue at a time when mitral valve closure would normally have occurred owing to the development of left ventricular tension. The mechanism for this delayed closure is not clear. It might have reflected an increase in left atrial pressure, agreeing with previous observations of the interval $Q$ to the onset of the first heart sound in mitral stenosis. ${ }^{10}$ Its presence would also be compatible with the finding of an accentuation of the amplitude of the mid-diastolic murmur of mitral stenosis in the early systolic period, particularly when the previous RR interval was short. ${ }^{11} \mathrm{~A}$ second factor may be the action of restoring forces responsible for the early phase of ventricular filling which imparts momentum to the blood, which in turn counteracts tension generated early in ventricular systole. Our results show this prolongation of filling to be a generalised phenomenon, independent of the aetiology of the heart disease, and suggest that it plays an important part in maintaining stroke volume when ventricular rate is rapid. Finally, its presence also indicates the difficulties that may arise in defining the phases of the cardiac cycle when ventricular filling, usually considered a diastolic event, occurs exclusively within the pre-ejection period, often described as a "systolic" time interval.

It was also possible to investigate factors determining the timing of mitral valve opening, itself dependent on the interval $\mathrm{QA}_{2}$, since isovolumic relaxation time remained virtually constant with the changing $R R$ interval. Variation of the $R R$ interval might be expected to influence $\mathrm{QA}_{2}$ in at least two ways. A long preceding $R R$ interval, by prolonging filling time, might be expected to increase stroke volume and thus to prolong ejection time. ${ }^{12} \mathrm{At}$ the same time variation in RR interval alters the strength of a subsequent ventricular contraction, the Bowditch and Woodworth effects. ${ }^{13}$ In atrial fibrillation in man prolongation of the RR interval immediately before the beat in question leads to an increase in the force of ventricular contraction $^{12}$ and thus in the absence of any other change to a reduction in $\mathrm{QA}_{2}$ interval. ${ }^{14}$ is Although these effects might be expected to counterbalance one another, our own and previous results ${ }^{16}$ strongly suggest that the effect on filling is considerably more important than any change in inotropic state. In the absence of mitral valve disease, reduction in the previous $R R$ interval to values of $600 \mathrm{~ms}$ or less led to a concomitant fall in $\mathrm{QA}_{2}$ and ejection times, whereas variation at $R R$ intervals greater than this value had little effect. This dependence on $R R$ interval, and hence on filling time, is incompatible with the Bowditch or Woodworth effects, which operate over the entire range of $R R$ intervals seen in clinical atrial fibrillation, ${ }^{17} 18$ but is entirely explicable by the normal dynamics of left ventricular filling. Since the rapid filling period at rest is virtually complete (in the absence of inflow obstruction) within $100 \mathrm{~ms}^{8}$ any further prolongation merely, takes in the period of diastasis and so is unaccompanied by further increase in ventricular volume. This suggestion is confirmed by prolongation of the range of dependence on $R R$ interval in patients with mitral stenosis, in whom rapid ventricular filling is lost with continuation of the volume increase into mid-diastole. Of the subdivi- 
sions of $\mathrm{QA}_{2}$, ejection time varied in a parallel manner with the changing preceding $R R$ interval, compatible with alterations in stroke volume. The pre-ejection period increased as preceding cycle length fell, but this was entirely explicable on the basis of the delay in mitral valve closure previously described.

Thus a simple combination of echocardiographic, phonographic, and mechanocardiographic techniques may be used to show fundamental physiological changes in the heart occurring continuously with varying pulse intervals in patients with atrial fibriallation. In particular, the time available for ventricular filling is very sensitive to the early onset of the succeeding systole, whereas that available for ejection is substantially preserved and shows much less variation. Nevertheless, mechanisms to maintain filling time exist when the RR interval is short. Mitral valve closure may be delayed by $20-30 \mathrm{~ms}$ and the duration of the filling period of the succeeding beat prolonged by up to $40 \mathrm{~ms}$ by earlier mitral valve opening. Although these time intervals may appear short, when taken together they constitute an appreciable proportion of the $100 \mathrm{~ms}$ required for the normal rapid filling period. This erosion of filling time may, nevertheless, be a major determinant of cardiac performance in atrial fibrillation, particularly in mitral stenosis when ventricular rate is fast. We suggest that if the effects of drugs or other physiological manoeuvres are to be investigated in patients with atrial fibrillation their diastolic actions as well as their effects on systolic function or heart rate should be taken into account. The predictable relations established here may provide the basis for a simple and non-invasive method by which this could be done.

The work was supported by grants from the British Council and Croucher Foundation.

\section{References}

1 Garrod AH. On some points connected with the circulation of blood, arrived at from a study of the sphygmograph-trace. Proc $R$ Soc Lond 1874; 23: 140-51.

2 Wiggers CJ. Studies on the consecutive phases of the cardiac cycle. I. The duration of the consecutive phases of the cardiac cycle and the criteria for their precise determination. Am F Physiol 1921; 56: 415-38.
3 Katz LN, Feil HS. Clinical observations on the dynamics of ventricular systole. I. Auricular fibrillation. Arch Intern Med 1923; 32: 672-92.

4 Weissler AM, Harris WS, Schoenfeld CD. Bedside technics for the evaluation of ventricular function in man. Am 7 Cardiol 1969; 23: 577-83.

5 Weissler AM, Peeler RG, Roehll WH Jr. Relationships between left ventricular ejection time, stroke volume, and heart rate in normal individuals and patients with cardiovascular disease. Am Heart f 1961; 62: 367-78.

6 Oldershaw PJ, Dawkins KD, Ward DE, Gibson DG. Diastolic mechanisms of impaired exercise tolerance in aortic valve disease $\mathrm{Br}$ Heart $\mathcal{F}$ 1983; 49: 568-73.

7 Weissler AM, Harris WS, Schoenfeld CD. Systolic time intervals in heart failure in man. Circulation 1968; 37: 149-59.

8 Hui WKK, Gibson DG. Mechanisms of reduced left ventricular filling rate in coronary artery disease. $\mathrm{Br}$ Heart $\mathcal{F}$ 1983; 50: 362-71.

9 Harris WS. Systolic time intervals in the noninvasive assessment of left ventricular performance in man. In: Mirsky I, Ghista DN, Sandler H, eds. Cardiac mechanics: physiological, clinical and mathematical considerations. New York and London: John Wiley, 1974: 233-92.

10 Wells $B$. The assessment of mitral stenosis by phonocardiography. Br Heart F 1954; 16: 261-6.

11 Criley JM, Hermer AJ. The crescendo presystolic murmur of mitral stenosis with atrial fibrillation. $N$ Engl f Med 1971; 285: 1284-7.

12 Greenfield JC Jr, Harley A, Thompson HK, Wallace AG. Pressure-flow studies in man during atrial fibrillation. $\mathcal{J}$ Clin Invest 1968; 47: 2411-21.

13 Woodworth RS. Maximal contraction, "staircase" contraction, refractory period, and compensatory pause of the heart. Am f Physiol 1902; 8: 213-49.

14 Weissler AM, Schoenfeld CD. Effect of digitalis on systolic time intervals in heart failure. Am F Med Sci 1970; 259: 4-20.

15 Shiner PT, Harris WS, Weissler AM. Effects of acute changes in serum calcium levels on the systolic time intervals in man. Am $\mathcal{F}$ Cardiol 1969; 24: 42-8.

16 van Dam I, van Zwieten G, Vogel JA, Meijler FL. Left ventricular (diastolic) dimensions and relaxation in patients with atrial fibrillation. Eur Heart $\mathcal{F} 1980 ; 1$ (suppl A):149-56.

17 Koch-Weser J, Blinks JR. The influence of the interval between beats on myocardial contractility. Pharmacol Rev 1963; 15: 601-52.

18 Gibson DG, Broder G, Sowton E. Effect of varying pulse interval in atrial fibrillation on left ventricular function in man. Br Heart f 1971; 33: 388-93. 